\title{
Effect of Nebivolol on Isolated Rat Bladder Strips Precontracted with Carbachol or Potassium Chloride
}

\author{
Hande Ozge Altunkaynak Camca \\ Department of Pharmacology, Gülhane Faculty of Pharmacy, University of Health Sciences Turkey, Ankara, Turkey.
}

Correspondence Author: Hande Ozge Altunkaynak Camca

E-mail: handeozgealtunkaynak.camca@sbu.edu.tr

Received: $03.05 .2020 \quad$ Accepted: 31.08 .2020

\begin{abstract}
Objective: One of the proposed mechanism mediating the vasorelaxant effect of nebivolol is based on its agonistic activity on beta-2 and/or beta- 3 adrenergic receptors. These receptors are also involved in the relaxation of urinary bladder. The aim of this study was to explore the ability of nebivolol to induce relaxation of the isolated rat bladder strip precontracted with cholinergic stimuli using carbachol or non-cholinergic stimuli using potassium chloride ( $\mathrm{KCl})$.

Methods: The isolated bladder strips were mounted in organ bath and contracted by $\mathrm{KCl}(40 \mu \mathrm{M})$ or carbachol $(1 \mathrm{mM})$ before the cumulative addition of nebivolol (0.0001-100 $\mu \mathrm{M})$. To investigate the role of beta-adrenergic receptors in the nebivolol-induced relaxant response, some bladder strips were incubated with propranolol $(1 \mu \mathrm{M})$ for $30 \mathrm{~min}$. Statistical significance was tested by Student's $t$-test. $p<0.05$ was considered to be statistically significant.

Results: Nebivolol elicited concentration-dependent relaxant response in the bladder strips precontracted with $\mathrm{KCl}$ or carbachol. Although the relaxant response to nebivolol in the bladder strips precontracted with carbachol was significantly inhibited by propranolol $(p<0.05)$, the nebivolol-induced relaxation failed to be inhibited by propranolol in the bladder strips precontracted with $\mathrm{KCl}$. The maximum relaxation in response to nebivolol was found to be significantly higher in the bladder strips precontracted with carbachol compared to that of $\mathrm{KCl}(p<0.05)$.

Conclusion: The findings of the present study indicate that beta-adrenergic receptors play role in the relaxant response of nebivolol in the isolated rat bladder strip precontracted with carbachol.
\end{abstract}

Keywords: Nebivolol, relaxation, carbachol, potassium chloride, bladder, rat

\section{INTRODUCTION}

Nebivolol is a selective beta-1 adrenergic receptor $\left(\beta_{1}-A R\right)$ blocker differently from conventional $\beta$-blockers because of its vasodilator and antioxidant properties $(1,2)$. Although the precise mechanisms by which nebivolol induces relaxation are not completely understood, there is increasing evidence showing that nitric oxide (NO) production by endotheliumdependent mechanisms including $\beta_{2}-$ and/or $\beta_{3}$-ARs are thought to be primarily responsible for the vasorelaxant effect of nebivolol $(3,4)$.

There is substantial evidence to indicate that $\beta$-ARs also involve in the relaxation of urinary bladder (5-7). Further evidence may come from the pharmacological studies which showed that the relaxant response to isoproterenol and other non-selective $\beta$-agonists is associated with $\beta_{2}$-and/or $\beta_{3}$-ARs in the rat bladder $(8,9)$.

The physiological voiding function of urinary bladder is controlled by the contraction of urinary bladder smooth muscle (10). This contraction is primarily elicited by muscarinic receptor stimulation by acetylcholine released from parasympathetic nerve endings $(10,11)$. However, under pathophysiological conditions, both cholinergic and non-cholinergic stimulus play role in the bladder contraction (12).

The present study was designed to investigate the effect of nebivolol on the isolated rat urinary bladder precontracted with either cholinergic or non-cholinergic stimulus induced by carbachol or potassium chloride $(\mathrm{KCl})$, respectively.

\section{METHODS}

\subsection{Drugs and Chemicals}

Nebivolol hydrochloride, carbachol and propranolol hydrochloride were obtained from Sigma-Aldrich (USA). All compounds were dissolved in distilled water except nebivolol. Nebivolol was dissolved in dimethylsulphoxide and the final concentration of the solvent in the organ bath was less than $0.01 \%(v / v)$. 


\subsection{Experimental Animals}

Experimental protocols were approved by KOBAY DHL Inc. Ethical Committee for Experimental Research on Animals (Approval date and number: 14.01.2020/451). Male SpragueDawley rats $(250-300 \mathrm{~g}, \mathrm{n}=6$ ) were used in this study. The rats were housed in cages and were allowed ad libitum access to standard laboratory diet and tap water.

\subsection{Experimental Design}

The anesthetized rats were sacrificed by cervical dislocation. Thereafter, the urinary bladder was rapidly excised and placed in Krebs-Henseleit solution (composition in $\mathrm{mM}$ : $\mathrm{NaCl}, 118 ; \mathrm{KCl}, 4.7 ; \mathrm{MgSO} 4 \bullet 7 \mathrm{H} 2 \mathrm{O}, 1.2 ; \mathrm{KH}_{2} \mathrm{PO}_{4}, 1.2 ; \mathrm{CaCl}_{2}$, 2.5; $\mathrm{NaHCO}_{3}, 25$; and glucose, 11). The bladder was cleaned from surrounding adjacent adipose and soft connective tissue. Afterwards, the upper most dome and the lower trigone area were removed and the remaining body of the bladder was sliced longitudinally into approximately $2 \times 10$ $\mathrm{mm}$ strips. The isolated bladder strips were placed in organ bath chambers attached to force displacement that were connected to a computer for isometric force recording. The Krebs-Henseleit solution in the bath was continuously aerated by mixture of $95 \% \mathrm{O}_{2}$ and $5 \% \mathrm{CO}_{2}$, and maintained at $37^{\circ} \mathrm{C}$. Resting tension of strips was set to $1 \mathrm{~g}$ and allowed to equilibrate for 60 minutes with replacing fresh KrebsHenseleit solution every $15 \mathrm{~min}$. To investigate whether the relaxant effect of nebivolol may differ in bladder strips due to distinct precontractile stimulus, carbachol for muscarinic receptor activation or $\mathrm{KCl}$ for membrane depolarization was used for this purpose. After the equilibration period, nebivolol (0.0001-100 $\mu \mathrm{M})$ was cumulatively added to organ bath to obtain cumulative concentration response curves (CCRCs) of the bladder strips precontracted with $\mathrm{KCl}(40 \mathrm{mM}$, $\mathrm{n}=6)$ or carbachol $(1 \mu \mathrm{M}, \mathrm{n}=5)$ and served as controls. To test the role of $\beta$-ARs in this response, some bladder strips were incubated for $30 \mathrm{~min}$ with propranolol (non-selective $\beta-A R$ antagonist, $1 \mu \mathrm{M})$.

\subsection{Statistical Analysis}

Data are expressed as mean \pm standard error of the mean (SEM). Relaxation is expressed as the percentage of the contraction caused by $\mathrm{KCl}$ or carbachol. Efficacy of nebivolol was expressed as maximum relaxation $\left(\mathrm{E}_{\max }\right)$. The analysis was performed using the statistical software package (GraphPad Prism, USA). Statistical significance was tested by Student's t-test. Differences were considered to be statistically significant when $p<0.05$.

\section{RESULTS}

Nebivolol elicited a concentration dependent relaxation in the $\mathrm{KCl}$-precontracted bladder strips $(n=6)$. However, there is no significant inhibition in the nebivolol-induced relaxation by the presence of propranolol (Figure 1, $p>0.05$ ).

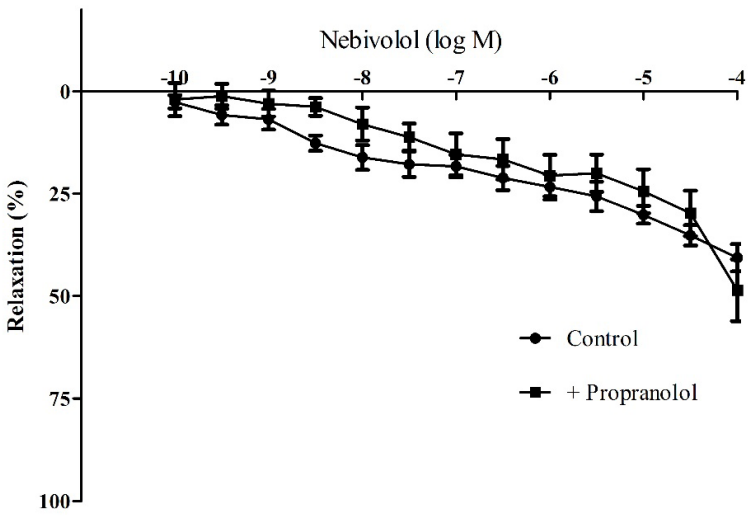

Figure 1. Nebivolol-induced relaxation in the rat bladder strips precontracted with $\mathrm{KCl}(40 \mathrm{mM})$ in the absence (Control, $n=6)$ or presence of propranolol (+Propranolol, $1 \mathrm{mM}, n=5$ )

Nebivolol also caused relaxation in the carbacholprecontracted bladder strips in a concentration-dependent manner $(n=5)$. Additionally, the relaxant response to nebivolol was significantly inhibited by the presence of propranolol in the isolated bladder strips (Figure 2, $p<0.05$ ).

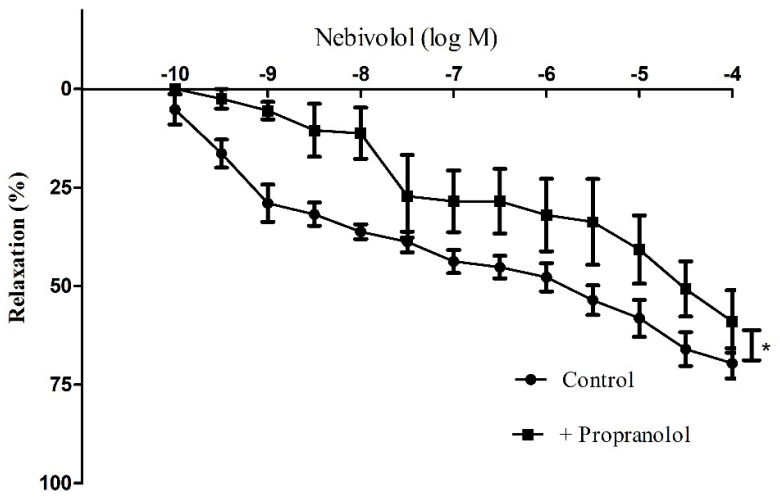

Figure 2. Nebivolol-induced relaxation in the rat bladder strips precontracted with carbachol $(1 \mu \mathrm{M})$ in the absence (Control, $n=5)$ or presence of propranolol (+Propranolol, $1 \mathrm{mM}, n=4)$. $p<0.05$ vs control

The maximum relaxation $\left(\mathrm{E}_{\max }\right)$ in response to nebivolol was significantly higher when bladder strips were precontracted with carbachol than that of $\mathrm{KCl}$ (Figure 3, $p<0.05$ ).

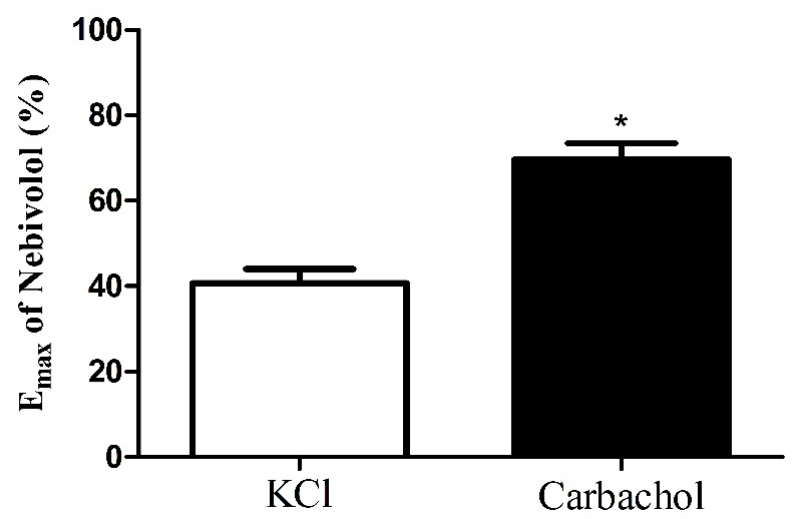

Figure 3. $E_{\max }$ of nebivolol in the rat bladder strips precontracted with $\mathrm{KCl}(40 \mathrm{mM}, n=6)$ or carbachol $(1 \mu \mathrm{M}, n=5)$. $p<0.05$ vs $\mathrm{KCl}$ 


\section{DISCUSSION}

In the present study, the relaxation induced by nebivolol was concentration-dependent both in the $\mathrm{KCl}$-precontracted or carbachol-precontracted isolated bladder strips. However, this response to nebivolol was found to be more efficacious when the precontracted stimulus was carbachol instead of $\mathrm{KCl}$. Additionally, the concentration-dependent relaxant effect of nebivolol was inhibited by propranolol in the bladder strips precontracted with carbachol.

As mentioned before, the contractions in the bladder evoked by carbachol or $\mathrm{KCl}$ indicate cholinergic and non-cholinergic stimulus, respectively. The present data showing a higher efficacy of nebivolol in the bladder strips precontracted with carbachol indicate that distinct mechanisms involve in the strength of precontraction contribute to differences in the ability of nebivolol to induce relaxation. The mechanisms responsible for the differences will be investigated in future studies, but one possible explanation of these findings is that different calcium sources play role in the contractile response to various stimulus in the bladder smooth muscle (13). Although the $\mathrm{KCl}$-induced contraction mainly depends on entry of calcium from extracellular sources, both calcium from extracellular sources and release from sarcoplasmic reticulum mediate the contractile response to carbachol in the bladder smooth muscle (13). Nebivolol has been reported to possess an inhibitor action on the both calcium sources and this may partly explain the question why nebivolol is more efficacious in the bladder strips precontracted with carbachol $(14,15)$.

The $\beta$-ARs are thought to be involved in the relaxation of the urinary bladder smooth muscle $(16,17)$. For this reason, some bladder strips were incubated with propranolol before addition of nebivolol cumulatively to the organ bath in the present study. Interestingly, propranolol inhibited the relaxant response to nebivolol in the bladder strips precontracted with carbachol but failed to inhibit this response in the $\mathrm{KCl}$ precontracted bladder strips. The present data support the hypothesis that $\beta$-ARs are involved in the nebivolol-mediated relaxation in the isolated urinary bladder strips precontracted with a cholinergic stimuli. However, the contribution of each subtype (especially $\beta_{2}$ - and $\beta_{3}$-ARs) in this action is needed to be elucidated by further studies.

The present data are in line with a previous study showing nebivolol-induced relaxation in the rat bladder strips precontracted with $\mathrm{KCl}(18)$. In the same study, it has also been shown that this response to nebivolol was insensitive to SR 59230A ( $\beta_{3}$-AR antagonist) (18). However, to the best of knowledge, there is no study evaluating the effects of nebivolol on the bladder strips precontracted with carbachol.

As previously mentioned, NO has been shown to be involved in the nebivolol-mediated vasorelaxation through endothelial $\beta_{2}-$ and/or $\beta_{3}$-ARs $(3,4)$. Additionally, NO has also been reported to play a role in the relaxation of isolated trigonal and urethral preparations (19). However, in the human detrusor smooth muscle, it has been found that the relaxation induced by $\beta-A R$ agonist might not relate with $\mathrm{NO}$ release (20). Although not addressed in the present study, the contribution of NO in the bladder relaxation through nebivolol is still question.

In conclusion, the present results show that nebivolol could produce more efficacious relaxation in the rat bladder strips precontracted with carbachol than that of $\mathrm{KCl}$. Additionally, these findings suggest that relaxation elicited by nebivolol is involved in the $\beta$-ARs in the isolated rat urinary bladder strips precontracted with a cholinergic stimuli.

\section{Acknowledgement}

I would like to thank to Zeynep Elif Yesilyurt (PhD student at Ankara University Faculty of Pharmacy, Department of Pharmacology) for technical support.

\section{REFERENCES}

[1] Olawi N, Krüger M, Grimm D, Infanger M, Wehland M. Nebivolol in the treatment of arterial hypertension. Basic Clin Pharmacol Toxicol 2019; 125 (3): 189-201.

[2] Cicero AFG, Kuwabara M, Borghi C. A critical review of nebivolol and its fixed-dose combinations in the treatment of hypertension. Drugs 2018; 78 (17): 1783-1790.

[3] Broeders MA, Doevendans PA, Bekkers BC, Bronsaer R, van Gorsel E, Heemskerk JW, Egbrink MG, van Breda E, Reneman RS, van Der Zee R. Nebivolol: a third-generation beta-blocker that augments vascular nitric oxide release: endothelial beta(2)-adrenergic receptor-mediated nitric oxide production. Circulation 2000; 102 (6): 677-684.

[4] de Groot AA, Mathy MJ, van Zwieten PA, Peters SL. Involvement of the beta3 adrenoceptor in nebivolol-induced vasorelaxation in the rat aorta. J Cardiovasc Pharmacol 2003; 42 (2): 232-236.

[5] Aizawa N, Homma Y, IgawaY. Effects of mirabegron, a novel $\beta 3$ adrenoceptor agonist, on primary bladder afferent activity and bladder microcontractions in rats compared with the effects of oxybutynin. Eur Urol 2012; 62 (6): 1165-1173.

[6] Igawa Y, Schneider T, Yamazaki Y, Tatemichi S, Homma Y, Nishizawa O, Michel MC. Functional investigation of $\beta$ adrenoceptors in human isolated detrusor focusing on the novel selective $\beta 3$-adrenoceptor agonist KUC-7322. Naunyn Schmiedebergs Arch Pharmacol 2012; 385 (8): 759-767.

[7] Propping S, Newe M, Lorenz K, Wirth MP, Ravens U. $\beta$-Adrenoceptor-mediated relaxation of carbachol-precontracted mouse detrusor. Urol Int 2015; 95 (1): 92-98.

[8] Oshita M, Hiraoka Y, Watanabe,Y. Characterization of betaadrenoceptors in urinary bladder: comparison between rat and rabbit. Br J Pharmacol 1997; 122 (8): 1720-1724.

[9] Yamazaki Y, Takeda H, Akahane M, Igawa Y, Nishizawa O, Ajisawa Y. Species differences in the distribution of beta-adrenoceptor subtypes in bladder smooth muscle. Br J Pharmacol 1998; 124 (3): 593-599.

[10] Visser AJ, van Mastrigt R. The role of intracellular and extracellular calcium in mechanical and intracellular electrical activity of human urinary bladder smooth muscle. Urol Res 2000; 28 (4): 260-268.

[11] Obara K, Suzuki S, Shibata H, Yoneyama N, Hamamatsu S, Yamaki F, Higai K, Tanaka Y. Noradrenaline-induced relaxation 
of urinary bladder smooth muscle is primarily triggered through the $\beta 3$-adrenoceptor in rats. Biol Pharm Bull 2019; 42 (5): 736-743.

[12] Michel MC, Sand C. Effect of pre-contraction on $\beta$-adrenoceptormediated relaxation of rat urinary bladder. World J Urol 2009; 27 (6): 711-715.

[13] Levin RM, Whitbeck C, Sourial MW, Tadrous M, Millington WR. Effects of dextromethorphan on in vitro contractile responses of mouse and rat urinary bladders. Neurourol Urodyn 2006; 25 (7): 802-807.

[14] Tan Z, Xiao Z, Wei J, Zhang J, Zhou Q, Smith CD, Nani A, Wu G, Song LS, Back TG, Fill M, Chen SR. Nebivolol suppresses cardiac ryanodine receptor-mediated spontaneous $\mathrm{Ca} 2+$ release and catecholaminergic polymorphic ventricular tachycardia. Biochem J 2016; 473 (22): 4159-4172.

[15] Cseplo P, Vamos Z, Ivic I, Torok O, Toth A, Koller A. The beta-1receptor blocker nebivolol elicits dilation of cerebral arteries by reducing smooth muscle [Ca2+]i. PLoS One 2016; 11 (10): e0164010.
[16] Uchida H, Shishido K, Nomiya M, Yamaguchi O. Involvement of cyclic AMP-dependent and - independent mechanisms in the relaxation of rat detrusor muscle via beta-adrenoceptors. Eur J Pharmacol 2005; 518 (2-3):195-202.

[17] Masunaga K, Chapple CR, McKay NG, Yoshida M, Sellers DJ. The $\beta 3$-adrenoceptor mediates the inhibitory effects of $\beta$-adrenoceptor agonists via the urothelium in pig bladder dome. Neurourol Urodyn 2010; 29 (7): 1320-1325.

[18] Frazier EP, Michel-Reher MB, van Loenen P, Sand C, Schneider T, Peters SL, Michel MC. Lack of evidence that nebivolol is a $\beta_{3^{-}}$ adrenoceptor agonist. Eur J Pharmacol 2011; 654 (1): 86-91.

[19] Persson K, Andersson KE. Nitric oxide and relaxation of pig lower urinary tract. Br J Pharmacol 1992; 106 (2):416-422.

[20] Otsuka A, Shinbo H, Matsumoto R, Kurita $Y$, Ozono S. Expression and functional role of beta-adrenoceptors in the human urinary bladder urothelium. Naunyn Schmiedebergs Arch Pharmacol 2008; 377 (4-6): 473-481. 